\title{
Sobrepeso, obesidad y niveles de presión arterial en niños de nivel 5 de jardines de infantes públicos de Montevideo: prevalencia y factores asociados
}

\author{
María Isabel Bove*, Gustavo Giachetto ${ }^{\dagger}$, Raúl Ramírez, Caren Zelmonovich§, \\ Valentina Guillermo§, Leticia Klaps§, Adriana Iturraldeף, Fabiana Peregalli§, \\ Daniel Bia**, Yanina Zócaloł†
}

\section{Resumen}

Introducción: en Uruguay, en los últimos años, se ha constatado un incremento en la prevalencia de sobrepeso y obesidad. La evidencia científica ha demostrado que las condiciones en la primera infancia son determinantes.

Objetivo: determinar la prevalencia de sobrepeso, obesidad y los niveles de presión arterial (PA) braquial e identificar factores de riesgo asociados en niños de nivel 5 que concurren a jardines públicos de Montevideo.

Método: estudio transversal (7/2016-6/2017) en una muestra representativa de 771 niños de nivel 5 de jardines públicos de Montevideo. Se relevó historia y comportamiento alimentario, actividad física, hábitos de sueño, peso y talla materna. En cada niño se registró antropometría y PA braquial.

Resultados: la prevalencia de sobrepeso u obesidad fue 40,6\% (IC95\%: 37,4-44,3), obesidad 16,5\% (IC95\%: 13,9-19,1) y obesidad abdominal 12,9\% (IC95\%: 11,0-15,8). Reportaron sobrepeso 29,4\% de las madres y obesidad 21,9\%. Se observó sedentarismo en $58,3 \%$, exposición prolongada a pantallas en $60,4 \%$ y escasas horas de sueño en $40,9 \%$. El consumo frecuente de alfajores, obleas y bizcochos en el desayuno, de embutidos entre semana, la preferencia de alimentos con publicidad, y el reporte de sobrepeso u obesidad materna se asociaron con obesidad infantil. Los niños con sobrepeso, obesidad u obesidad abdominal presentaron mayores niveles de PA braquial.

Conclusiones: la prevalencia de sobrepeso y obesidad en niños de nivel 5 de jardines públicos de Montevideo es elevada. El sobrepeso, la obesidad materna y los hábitos en relación con la alimentación, actividad física y sueño se asocian con su presencia.

\footnotetext{
* Dra. en Nutrición. Universidad de Granada, España. Investigadora de la Universidad Católica del Uruguay.

† Médico, pediatra. Profesor director de Clínica Pediátrica "C", Facultad de Medicina, Universidad de la República.

¥ Estadístico. Profesor de la Universidad Católica del Uruguay y de la Universidad de la República.

$\S$ Licenciada en Nutrición. Maestría en Nutrición, Universidad Católica del Uruguay.

ๆl Médica, pediatra. Exprofesora adjunta de Clínica Pediátrica "C", Facultad de Medicina, Universidad de la República. Maestrando en Nutrición,

Universidad Católica del Uruguay.

** Doctor en Ciencias Biológicas. Prof. Agreg. Depto. Fisiología, director del Centro Universitario de Investigación, Innovación y Diagnóstico Arterial (CUiiDARTE), Facultad de Medicina, Universidad de la República.

†† Médica. Doctora en Ciencias Biomédicas. Profesora adjunta, Depto. Fisiología. Directora del Centro Universitario de Investigación, Innovación y Diagnóstico Arterial (CUiiDARTE), Facultad de Medicina, Universidad de la República.

Universidad Católica del Uruguay. Clínica Pediátrica "C", Facultad de Medicina, Universidad de la República. Centro Universitario de Investigación, Innovación y Diagnóstico Arterial (CUiiDARTE). Departamento de Fisiología, Facultad de Medicina, Universidad de la República.

Correspondencia: Dra. María Isabel Bove. Correo electrónico: isabelbove.uru@gmail.com

Conflicto de intereses: no existen conflicto de intereses para reportar. El proyecto de investigación fue financiado por el Fondo Sectorial de Primera Infancia ANII, UCC, UNICEF (FSPI_X_2015_1_108509 y FSPI_X_2015_1_108484).

El protocolo de estudio fue autorizado por el Consejo de Educación Inicial y Primaria, por las autoridades de los centros educativos participantes y por el Comité de Ética de la Universidad Católica del Uruguay.

Recibido: 18/7/19

Aprobado: 23/3/20
} 
Palabras clave: Sobrepeso

Obesidad pediátrica

Niño

Hipertensión

Prevalencia

Factores de riesgo

$\begin{array}{ll}\text { Key words: } & \text { Overweight } \\ & \text { Pediatric obesity } \\ & \text { Child } \\ & \text { Hypertension } \\ & \text { Prevalence } \\ & \text { Risk factors }\end{array}$

Muestra

Para el diseño y cálculo muestral se utilizó la base de datos del padrón elaborado por la Unidad de Estadística del Consejo de Educación Inicial y Primaria (CEIP, 2015). La unidad de muestreo fue el grupo de alumnos del jardín, clasificados según la jurisdicción de Montevideo: centro, este, oeste. El diseño fue probabilístico, bi-etápico y estratificado, proporcional a la cantidad de alumnos de cada centro. Se seleccionaron 24 jardines de infantes (26 grupos de nivel 5). Se incluyeron en la muestra 771 niños y niñas. Usando las variables sexo y jurisdicción, se posestratificó la muestra para realizar la calibración de los ponderadores originales. El tamaño muestral conduce a un error máximo global de 3,6\%.

\section{Recolección de datos y variables}

Para el trabajo de campo se conformó un equipo de estudiantes y licenciados en Nutrición, médicos y enfermeros, previamente capacitados siguiendo las pautas nacionales de modo de estandarizar la toma de las medidas antropométricas. Las base de datos fueron las entrevistas estructuradas a padres o tutores y la antropometría de los niños.

\section{Entrevista estructurada}

Mediante entrevista a las madres se relevó información acerca de historia y comportamiento alimentario, actividad física, hábitos de sueño, peso y talla materna actual. En la historia y comportamiento alimentario del niño se indagó número de comidas diarias, alimentos consumidos en el desayuno y frecuencia de consumo de frutas, verduras, pescado, refrescos, golosinas, snacks, nuggets, hamburguesas y frankfurters o "panchos". Se relevaron algunos determinantes de la dieta, como manejo de dinero por parte del niño para comprar comida; disponibilidad de frutas, verduras y golosinas; utilización de alimentos como recompensa o castigo; consumo durante el juego; recuerdo de mensajes de publicidades de alimentos, y preferencia de alimentos promocionados en televisión o que adjuntan juguetes. Se indagó el tipo, frecuencia y duración de actividad física en el jardín de infantes, centros deportivos y durante el ocio, y el desplazamiento (a pie o en bicicleta), mediante for- 
mularios previamente validados ${ }^{(8)}$. Se estimó el tiempo total de actividad física diaria, de lunes a viernes, $y$ se consideró que el niño era activo cuando reportaban que realizaba 60 minutos o más de actividad física diaria $^{(9)}$. En el tiempo libre, se analizó el tiempo de exposición a pantalla (televisor, celular, DVD, video, juegos electrónicos, computadora). Se consideró que el niño superó el límite de horas de exposición a pantalla cuando a los 5 y 6 años se reportaba que permanecía 1 o 2 horas diarias, respectivamente, según las recomendaciones de la Academia Americana de Pediatría $^{(10,11)}$. Se evaluaron otras actividades sedentarias durante el tiempo libre, como el juego pasivo (observación, lectura, juego con muñecas o autitos, pintura). Se evaluaron los hábitos de sueño en la noche (hora de acostarse y levantarse) de lunes a viernes. Se consideró que cumplía con las horas de sueño recomendadas si se reportaba que dormía a los 5 y 6 años $\geq 10$ horas $y \geq 9$ horas, respectivamente ${ }^{(12)}$.

\section{Antropometría}

El peso y la talla materna al momento de la entrevista se recabaron por autorreporte. Se calculó el índice de masa corporal (IMC): peso $(\mathrm{kg}$ ) dividido por el cuadrado de la estatura (metros). Se consideró sobrepeso cuando el IMC se encontraba entre 25 y $29,9 \mathrm{~kg} / \mathrm{m}^{2}$, y obesidad cuando el IMC $\geq 30 \mathrm{~kg} / \mathrm{m}^{2(13)}$. En el caso de los niños, el registro de la talla se realizó de pie, de espaldas al tallímetro, sin zapatos, con la menor ropa posible y sin sujeción del cabello. Se utilizaron estadiómetros $\mathrm{Seca}^{\mathbb{B}}$ (modelo 213, precisión $1 \mathrm{~mm}$ ). El registro de peso se hizo de pie, con el niño inmóvil, mediante balanzas Seca ${ }^{\circledR}$ (modelos 843 y 841 , precisión $\left.0,1 \mathrm{~kg}\right)^{(14,15)}$. Se midió la circunferencia de la cintura $(\mathrm{CC})$ en posición erguida, con el torso descubierto, los brazos relajados y paralelos al tronco mediante una cinta Seca ${ }^{\circledR}$ (modelo 201, precisión $1 \mathrm{~mm}$ ), colocada a una distancia media entre el borde inferior de la última costilla y el borde superior de la cresta ilíaca ${ }^{(16)}$. Cada una de las medidas fue realizada por duplicado por técnicos diferentes y se registró el promedio. Las diferencias máximas admitidas entre la primera y la segunda medición fueron $\pm 0,30 \mathrm{~g}$, $\pm 0,30 \mathrm{~cm} \mathrm{y} \pm 0,50 \mathrm{~cm}$ para peso, talla y CC, respectivamente. Se calculó el score Z de IMC/edad (IMC/E), peso/edad (P/E) y peso/talla (PT/E) mediante el software AnthroPlus v1.0.2 de la Organización Mundial de la Salud (OMS). Se excluyeron los puntajes Z IMC/E menores a -5 y mayores a 5 , y de P/E y PT/E menores a -6 y mayores a 6. Para evaluar sobrepeso y obesidad se utilizó el IMC/E. Se consideró sobrepeso $\mathrm{Z}$ mayor a 1 desvío estándar (DE), obesidad $Z$ mayor a $2 \mathrm{DE}$ y obesidad abdominal cuando la CC fue mayor al percentil 90 de los patrones de referencia ${ }^{(13-16)}$.

\section{Presión arterial periférica (braquial)}

Los registros fueron realizados por integrantes de CUiiDARTE que asistieron a cada jardín en horario de clases. En cada medición se determinó la PA sistólica (PAS) y diastólica (PAD) braquial por método auscultatorio (presurómetro anaeroide y estetoscopio) y oscilométrico (sistema semiautomático Hem-4030, Omron Inc., Estados Unidos; sistema automático Mobil-O-Graph, IEM-GmbH, Alemania). Los registros se realizaron en un ambiente cerrado, iluminado, silencioso y con temperatura cálida controlada, al menos $30 \mathrm{mi}-$ nutos después de realizar ejercicio o ingerir alimentos, con el niño sentado durante 5-10 minutos. Para las mediciones se utilizaron manguitos con las medidas internacionales recomendadas. Se obtuvieron al menos tres mediciones en cada brazo, con un intervalo mínimo de 3 minutos entre cada medición. Para el presente estudio se consideró el promedio de los registros auscultatorios; en caso de no haberse podido obtener mediciones auscultatorias confiables, se consideró el promedio de los registros oscilométricos. En función de la edad, el sexo y el percentil de altura se consideraron normales cifras de PAS o PAD $<$ percentil 90, normal-alto $\geq$ percentil $90 \mathrm{y}<$ percentil 95 , e hipertensión arterial $\geq$ percentil $95^{(17,18)}$.

\section{Factores de riesgo}

Se analizó la relación entre sobrepeso u obesidad en niños de nivel 5 con actividad física, horas de exposición a pantallas y de sueño, hábitos y composición de la alimentación e IMC materno aproximado.

\section{Aspectos éticos}

El protocolo de estudio fue autorizado por el CEIP, por las autoridades de los centros educativos participantes y por el Comité de Ética de la Universidad Católica del Uruguay. Se solicitó consentimiento de padres, madres o tutores.

\section{Análisis estadístico}

Las variables cualitativas se describen en frecuencias absolutas y relativas, las cuantitativas en medidas de tendencia central y dispersión (media, DE). Para la comparación de proporciones se utilizó el test de chi cuadrado y para la comparación de medias y varianzas la prueba $t$ de Student y el análisis de varianza de ANOVA. Para la estimación de riesgo se aplicó un modelo de regresión logística binaria. Se estimó la razón de probabilidades (odds ratio, OR), informándose su nivel medio e intervalo de confianza del 95\% (IC95\%). Se consideró estadísticamente significativo una $\mathrm{p}<0,05$. En el procesamiento de los datos se utilizó el programa SPSS Statistics Base V22.0. 


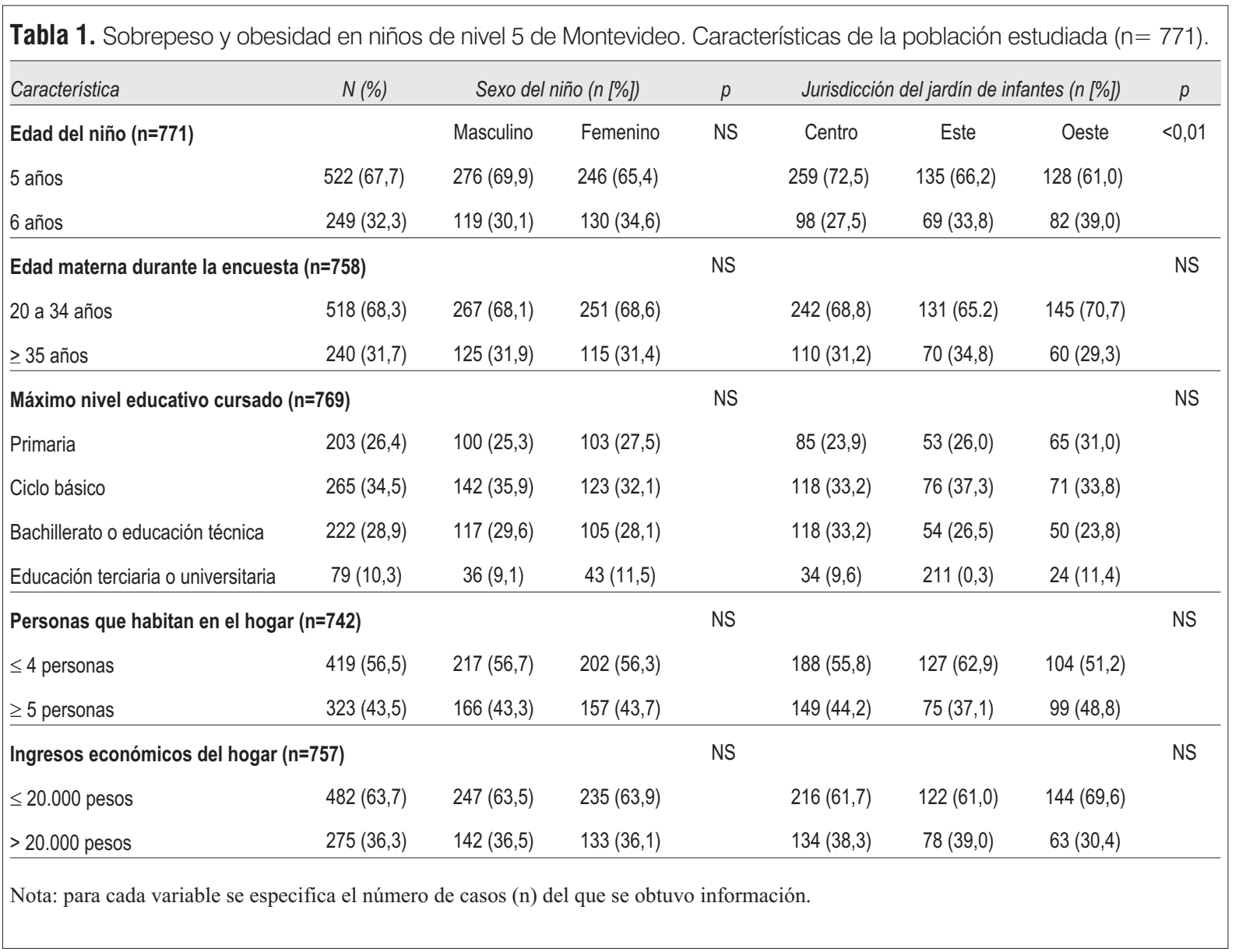

\section{Resultados}

En la tabla 1 se muestran algunas de las características de la población estudiada. Tenían 5 años el $67,7 \%$ de los niños, el resto había cumplido 6 años. Eran menores de 35 años el $68,3 \%$ de las madres y habían cursado educación primaria $26,4 \%$ y ciclo básico $34,5 \%$. La mayoría de los hogares estaban conformados por $\leq 4$ personas y tenían ingresos $\leq \$ 20.000$. No se observaron diferencias significativas en las características mencionadas según el sexo del niño y la jurisdicción del jardín de infantes.

La tabla 2 muestra la prevalencia de sobrepeso $\mathrm{u}$ obesidad. El 40,6\% (IC95\%: 37,4-44,3\%) presentó sobrepeso u obesidad; el 16,5\% (IC95\%: 13,9-19,1\%) obesidad, y el 12,9\% (IC95\%: 11,0-15,8\%) obesidad abdominal. No se observaron diferencias significativas en la distribución por sexo y jurisdicción.

La tabla 3 presenta los datos de actividad física, horas de pantalla y horas de sueño en los niños estudiados. El 61\% tenía clases de educación física en el jardín de infantes de 30 a 60 minutos, dos o más veces por semana. La frecuencia de clases de educación física fue significativamente menor en los niños que concurrían a los jar- dines de la jurisdicción centro. El 20,9\% acudía, además, a algún centro deportivo; la frecuencia de asistencia fue significativamente menor en niñas y en los de la jurisdicción oeste. Resultaron activos el 41,7\% de los niños. Excedían el tiempo recomendado de exposición a pantallas $60,4 \%$ y dormían menos que la cantidad de horas recomendadas $40,9 \%$, siendo esta última proporción significativamente mayor en aquellos procedentes de la región oeste. El promedio diario de actividad física fue 1 hora y media ( $95 \pm 88$ minutos), de exposición a pantallas más de 2 horas y media (155 \pm 119 minutos), y de tiempo sedentario más de 4 horas ( $265 \pm 155$ minutos); no observándose diferencias por sexo ni jurisdicción. El promedio diario de horas de sueño fue $9,8 \pm 1,2$ horas.

En la tabla 4 se muestran algunas características de la alimentación del niño y la prevalencia de sobrepeso u obesidad materna al momento de la entrevista por autorreporte. El 31,3\% no desayunaba, y entre los niños que lo hacían, la mayoría consumía leche y pan, casi la mitad consumía alfajores, galletitas rellenas o bizcochos, y un tercio yogurt o leche chocolatada. No consumían verduras y frutas a diario $77,1 \%$ y $50,3 \%$, respectivamente. El consumo de pescado resultó muy esporádico. La mayo- 
Tabla 2. Sobrepeso y obesidad en niños de nivel 5 de Montevideo. Prevalencia de sobrepeso, obesidad y obesidad abdominal según sexo y jurisdicción $(n=771)$.

\begin{tabular}{|c|c|c|c|c|c|c|c|c|}
\hline & \multicolumn{8}{|c|}{$\begin{array}{c}\text { Prevalencia } \\
n, \% \\
\text { (IC 95\%) }\end{array}$} \\
\hline & \multicolumn{2}{|c|}{ Sexo } & \multirow[t]{2}{*}{$p$} & \multicolumn{3}{|c|}{ Jurisdicción } & \multirow[t]{2}{*}{$p$} & \multirow[t]{2}{*}{ Total } \\
\hline & Varones $(n=395)$ & Niñas $(n=375)$ & & Centro $(n=278)$ & Este $(n=229)$ & Oeste $(n=265)$ & & \\
\hline Sobrepeso u obesidad & $\begin{array}{c}163,41,3 \\
(36,9-46,6)\end{array}$ & $\begin{array}{c}150,40,0 \\
(34,9-44,9)\end{array}$ & NS & $\begin{array}{c}140,39,2 \\
(33,4-44,9)\end{array}$ & $\begin{array}{c}90,44,1 \\
(37,6-50,6)\end{array}$ & $\begin{array}{c}83,39,7 \\
(33,8-45,9)\end{array}$ & NS & $\begin{array}{r}313,40,6 \\
(37,4-44,3)\end{array}$ \\
\hline Obesidad & $\begin{array}{c}63,15,9 \\
(12,4-19,7)\end{array}$ & $\begin{array}{c}64,17,1 \\
(13,1-20,8)\end{array}$ & & $\begin{array}{c}60,16,8 \\
(12,3-21,2)\end{array}$ & $\begin{array}{c}30,14,7 \\
(10,1-19,3)\end{array}$ & $\begin{array}{c}37,17,7 \\
(13,1-22,4)\end{array}$ & & $\begin{array}{r}127,16,5 \\
(13,9-19,1)\end{array}$ \\
\hline Obesidad abdominal & $\begin{array}{c}46,11,7 \\
(9,3-15,8)\end{array}$ & $\begin{array}{c}53,14,1 \\
(10,7-17,8)\end{array}$ & & $\begin{array}{c}37,10,4 \\
(6,8-13,9)\end{array}$ & $\begin{array}{c}28,13,7 \\
(9,2-18,2)\end{array}$ & $\begin{array}{c}34,16,2 \\
(11,7-20,7)\end{array}$ & & $\begin{array}{c}99,12,9 \\
(11,0-15,8)\end{array}$ \\
\hline
\end{tabular}

ría tomaba refrescos y consumía nuggets, "panchos" o fiambres tres o más días a la semana. Más del $80,0 \%$ de los niños recordaba mensajes de publicidad de alimentos y la mitad de ellos preferían aquellos que se publici$\tan$ en la televisión. La preferencia por estos alimentos fue significativamente mayor en las niñas. El 21,9\% de las madres presentaba obesidad por autorreporte al momento de la entrevista; la proporción fue significativamente mayor en las madres de los niños de los jardines de la jurisdicción centro.

En la tabla 5 se muestra la asociación observada de sobrepeso, obesidad y obesidad abdominal con los hábitos de sueño, actividad física y alimentación. Los niños que vivían en hogares con cuatro integrantes o menos presentaron mayor riesgo de obesidad en relación con aquellos procedentes de hogares más numerosos. El consumo de alfajores, obleas y bizcochos en el desayuno, de nuggets, "panchos" o fiambres en forma frecuente, y la preferencia de alimentos que traen juguetes se asociaron con mayor riesgo de obesidad a los 5 años (RR: 1,5, IC95\%: 1,06-2,3; RR: 1,6, IC95\%: 1,05-2,3; RR: 1,5, IC95\%: 1,01-2,1; respectivamente). El sobrepeso y la obesidad en la madre, según el IMC aproximado, se asoció con sobrepeso y obesidad en el niño. En esta serie no se observó asociación significativa con el hábito de no desayunar y la frecuencia de consumo de frutas, verduras y refrescos.

La tabla 6 presenta los niveles de PA y los niveles medios de percentil existente en los niños. El percentil de PA periférica (braquial) promedio según sexo, edad y talla resultó más alto para el conjunto de niños estudiados que lo esperado para una población referencia (PAS P60, PAD P62). Los niños con sobrepeso, obesidad y obesidad abdominal presentaron promedios significati- vamente más elevados de PAS y PAD (braquial) que el total de niños, tanto en términos absolutos como en nivel percentilar $(\mathrm{p}<0,001)$.

\section{Discusión}

El diseño y tamaño de la muestra, así como la metodología utilizada en el registro de las variables antropométricas y arteriales, constituyen las principales fortalezas de esta investigación que muestran cifras preocupantes de sobrepeso u obesidad en los niños de nivel 5 que concurrieron a los jardines de infantes públicos de Montevideo en los años 2016 y 2017. La OMS estima que a nivel mundial $18 \%$ de los niños y adolescentes de 5 a 19 años tiene exceso de peso $(>1 \mathrm{DE})$ y $7 \%$ obesidad $(>2$ $\mathrm{DE})^{(19)}$. La presente investigación reveló que el $24,3 \%$ de los niños de nivel 5 presentó exceso de peso (>1 DE) y $16,5 \%$ obesidad ( $>2 \mathrm{DE}$ ), es decir, la prevalencia resultó más del doble que la estimada a nivel mundial. En algunos países europeos se estima que el $20,8 \%$ de los niños menores de 9 años presenta exceso de peso y el $7 \%$ obesidad $^{(20)}$. La prevalencia de obesidad hallada en este estudio resultó superior a la observada en países europeos con mayor prevalencia, como España, que en el año 2015 comunicó 34,6\% de exceso de peso y 14,9\% de obesidad en niños de 6 años ${ }^{(19)}$. Poniendo el foco en la región, el $20 \%$ de los niños y adolescentes latinoamericanos de 0 a 19 años tienen exceso de peso y los países más afectados son los del Cono Sur y México ${ }^{(21,22)}$. Los guarismos del presente trabajo, comparados con Brasil, resultaron más elevados respecto al exceso de peso $(32,8 \%)$ y similares en relación con obesidad $(16,2 \%)$, pero inferiores a los de Chile, el país más afectado de la región, con una prevalencia de obesidad de $24,2 \%$ en niños de 5 a 6 años ${ }^{(23,24)}$. 


\begin{tabular}{|c|c|c|c|c|c|c|c|c|}
\hline & $N(\%)$ & Varones & Niñas & $p$ & Centro & Este & Oeste & $p$ \\
\hline \multicolumn{3}{|c|}{ Educación física en el jardín ( $n=771$ ) } & & NS & & & & $<0,01$ \\
\hline No tenían clases & $300(39,0)$ & $143(36,3)$ & $157(41,8)$ & & $191(53,7)$ & $70(34,3)$ & $39(18,6)$ & \\
\hline 1 día/semana & $205(26,6)$ & $113(28,7)$ & $92(24,5)$ & & $41(34,8)$ & $90(21,6)$ & $74(46,2)$ & \\
\hline$\geq 2$ días/semana & $265(34,4)$ & $138(35,0)$ & $127(33,8)$ & & $124(34,8)$ & $44(21,6)$ & $97(46,2)$ & \\
\hline \multicolumn{2}{|l|}{ Centro deportivo $(n=771)$} & & & $<0,01$ & & & $<0,05$ & \\
\hline No concurre & $600(79,1)$ & $279(72,3)$ & $321(86,1)$ & & $274(78,5)$ & $154(76,6)$ & $172(82,3)$ & \\
\hline Concurre 1 dia/semana & $33(4,3)$ & $22(5,7)$ & $11(2,9)$ & & $11(3,2)$ & $8(4,0)$ & $14(6,7)$ & \\
\hline Concurre $\geq 2$ días/semana & $126(16,6)$ & $85(22,0)$ & $41(11,0)$ & & $64(18,3)$ & $39(19,4)$ & $23(11,0)$ & \\
\hline \multicolumn{2}{|c|}{ Se traslada caminando al jardín $(n=750)$} & & & NS & & & & NS \\
\hline 0 día/semana & $152(20,3)$ & $80(20,7)$ & $72(19,8)$ & & $76(21,9)$ & $47(24,0)$ & $29(14,0)$ & \\
\hline 1-2 días/semana & $110(14,7)$ & $54(14,0)$ & $56(15,4)$ & & $54(15,6)$ & $28(14,3)$ & $28(13,5)$ & \\
\hline 3-4 días/semana & $343(45,7)$ & $191(49,4)$ & $152(41,9)$ & & $147(42,4)$ & $85(43,4)$ & $111(53,6)$ & \\
\hline 5 días/semana & $145(19,3)$ & $62(16,0)$ & $83(22,9)$ & & $70(22,2)$ & $36(18,4)$ & $39(18,8)$ & \\
\hline \multicolumn{2}{|l|}{ Traslado en bicicleta $(n=764)$} & & & NS & & & & NS \\
\hline 0 día/semana & $590(77,2)$ & $301(76,2)$ & $289(78,3)$ & & $280(79,1)$ & $1517(4,8)$ & $159(76,4)$ & \\
\hline 1 a 2 días/ semana & $124(16,2)$ & $65(16,5)$ & $59(16,0)$ & & $54(15,3)$ & $39(19,3)$ & $31(14,9)$ & \\
\hline 3 a 4 días/ semana & $27(3,5)$ & $17(4,3)$ & $10(2,7)$ & & $11(3,1)$ & $7(3,5)$ & $9(4,3)$ & \\
\hline 5 días/semana & $23(3,0)$ & $12(3,0)$ & $11(3,0)$ & & $9(2,5)$ & $5(2,5)$ & $9(4,3)$ & \\
\hline \multicolumn{9}{|c|}{ Nivel diario de actividad física $(n=667$ ) } \\
\hline$<60$ min por día & $389(58,3)$ & $188(55,6)$ & $201(61,1)$ & & $177(58,2)$ & $110(62,9)$ & $102(54,3)$ & \\
\hline$\geq 60$ min por día & $278(41,7)$ & $150(44,4)$ & $128(38,9)$ & & $127(41,8)$ & $65(37,1)$ & $86(45,7)$ & \\
\hline \multicolumn{9}{|l|}{ Horas pantalla/día ( $n=747$ ) } \\
\hline$>2$ & $451(60,4)$ & $234(60,5)$ & $217(60,3)$ & NS & $207(60.2)$ & $123(62,4)$ & $121(58,7>)$ & NS \\
\hline \multicolumn{9}{|c|}{ Horas de sueño nocturno/día $(n=760)$} \\
\hline No cumple con recomendación & $311(40,9)$ & $166(42,6)$ & $145(39,1)$ & NS & $152(43,3)$ & $59(29,4)$ & $100(48,1)$ & $<0,01$ \\
\hline Cumple con recomendación & $449(59,1)$ & $224(57,4)$ & $225(60,9)$ & & $199(56,7)$ & $142(70,6)$ & $108(51,9)$ & \\
\hline
\end{tabular}

Preocupa no solo las cifras elevadas, sino la tendencia acelerada al ascenso. En Uruguay, en el año 2000, el $26 \%$ de niños de 9 a 12 años presentaba exceso de peso y $9 \%$ obesidad $^{(25)}$. Cuatro años después, las cifras se mantenían estables ${ }^{(26)}$. Sin embargo, desde 2004 a 2017, según el presente trabajo y el desarrollado por la Comisión de Salud Cardiovascular en 2016, la prevalencia de obesidad infantil aumentó, ya que las cifras son significativamente mayores a edades más precoces ${ }^{(27)}$.
Hasta hace unos años la grasa corporal era considerada como un almacenamiento de energía, sin funciones hormonales ni metabólicas, y el exceso de grasa corporal en los niños se consideraba solo un problema estético o un obstáculo para el rendimiento físico, en lugar de un verdadero problema de salud ${ }^{(28)}$. La epidemia de sobrepeso y obesidad y su inicio temprano en la infancia hacen necesario considerar el exceso de grasa corporal como un factor de riesgo cardiovascular también en la edad 
Tabla 4. Sobrepeso y obesidad en niños de nivel 5 de Montevideo. Alimentación del niño y obesidad materna al momento de la entrevista.

\begin{tabular}{|c|c|c|c|c|c|c|c|c|}
\hline \multirow[t]{2}{*}{ Alimentación ( $N=768)$} & \multirow[t]{2}{*}{$N(\%)$} & \multicolumn{2}{|c|}{ Sexo del niño [n (\%)] } & \multirow[t]{2}{*}{$p$} & \multicolumn{3}{|c|}{ Jurisdicción [n (\%)] } & \multirow[t]{2}{*}{$p$} \\
\hline & & Varones & Niñas & & Centro & Este & Oeste & \\
\hline \multicolumn{9}{|l|}{ Número de comidas diarias } \\
\hline 4 & $528(68,8)$ & $279(71,0)$ & $249(66,4)$ & NS & $246(69,3)$ & $145(71,4)$ & $137(65,2)$ & NS \\
\hline 3 (no desayuna) & $240(31,3)$ & $114(29,0)$ & $126(33,6)$ & & $109(30,7)$ & $58(28,6)$ & $73(34,8)$ & \\
\hline \multicolumn{9}{|l|}{ Alimentos ingeridos en el desayuno } \\
\hline Leche & $660(85,8)$ & $343(87,1)$ & $317(84,5)$ & NS & $299(84,2)$ & $183(89,7)$ & $178(84,8)$ & NS \\
\hline Yogurt o leche chocolatada & $269(35,0)$ & $152(38,6)$ & $117(31,2)$ & $<0.05$ & $128(36,1)$ & $57(27,9)$ & $84(40,0)$ & $<0.05$ \\
\hline Pan & $610(79,4)$ & $320(81,2)$ & $290(77,5)$ & NS & $274(77,4)$ & $164(80,4)$ & $172(81,9)$ & NS \\
\hline $\begin{array}{l}\text { Alfajores, galletitas rellenas } \\
\text { o bizcochos }\end{array}$ & $368(47,9)$ & $182(46,2)$ & $186(49,6)$ & NS & $180(50,7)$ & $87(42,6)$ & $101(48,1)$ & NS \\
\hline \multicolumn{9}{|l|}{ Frecuencia de consumo de alimentos } \\
\hline No consumo diario de frutas & $387(50,3)$ & $210(53,3)$ & $177(47,2)$ & NS & $179(50,4)$ & $109(53,3)$ & $99(47,2)$ & NS \\
\hline No consumo diario de verduras & $590(77,1)$ & $302(77)$ & $288(77,2)$ & NS & $278(79,1)$ & $160(78,9)$ & $152(72,3)$ & NS \\
\hline $\begin{array}{l}\text { No consumo o consumo esporádico de } \\
\text { pescado }\end{array}$ & $537(70,6)$ & $278(71,3)$ & $259(69,8)$ & NS & $235(66,8)$ & $152(75,7)$ & $150(72,1)$ & NS \\
\hline Refrescos $\geq 3$ días por semana & $442(57,9)$ & $323(57,0)$ & $219(58,9)$ & NS & $195(55,7)$ & $124(61,0)$ & $123(58,5)$ & NS \\
\hline Golosinas $\geq 3$ días por semana & $242(32,0)$ & $118(30,1)$ & $124(33,1)$ & NS & $102(28,7)$ & $70(34,6)$ & $70(33,3)$ & NS \\
\hline $\begin{array}{l}\text { Nuggets, "panchos", fiambres: } \geq 3 \text { veces } \\
\text { por semana }\end{array}$ & $544(71,3)$ & $263(67,1)$ & $281(75,7)$ & NS & $256(73,1)$ & $141(69,5)$ & $147(70,0)$ & NS \\
\hline \multicolumn{9}{|l|}{ Condicionantes familiares } \\
\hline $\begin{array}{l}\text { Nunca u ocasionalmente se le ofrece } \\
\text { comida casera }\end{array}$ & $40(5,2)$ & $17(4,6)$ & $23(5,9)$ & NS & $18(5,1)$ & $8(3,9)$ & $14(6,8)$ & NS \\
\hline $\begin{array}{l}\text { Siempre o casi siempre se le ofrecen } \\
\text { alimentos mientras juega }\end{array}$ & $258(34,4)$ & $130(33,2)$ & $128(35,7)$ & NS & $105(29,7)$ & $69(33,8)$ & $84(40,0)$ & $<0,05$ \\
\hline $\begin{array}{l}\text { Frecuentemente se utilizan alimentos } \\
\text { como recompensa o castigo }\end{array}$ & $28(3,7)$ & $16(4,1)$ & $12(3,2)$ & NS & $10(2,9)$ & $7(3,4)$ & $11(5,3)$ & NS \\
\hline $\begin{array}{l}\text { El niño maneja dinero para comprar } \\
\text { comida }\end{array}$ & $82(10,8)$ & $38(9,7)$ & $44(11,9)$ & NS & $40(11,3)$ & $20(10,0)$ & $22(10,7)$ & NS \\
\hline \multicolumn{9}{|l|}{ Preferencia y publicidad (siempre o casi) } \\
\hline $\begin{array}{l}\text { Recuerda mensajes de publicidad sobre } \\
\text { alimentos }\end{array}$ & $625(81,3)$ & $307(77,9)$ & $318(84,8)$ & $<0,05$ & $293(82,5)$ & $163(79,9)$ & $169(80,4)$ & NS \\
\hline Prefiere alimentos publicitados en la TV & $330(43,3)$ & $155(39,3)$ & $175(47,4)$ & $<0,05$ & $150(43,0)$ & $84(41,4)$ & $96(45,8)$ & NS \\
\hline $\begin{array}{l}\text { Prefiere alimentos que adjuntan } \\
\text { juguetes }\end{array}$ & $520(67,9)$ & $266(67,5)$ & $254(68,3)$ & NS & $247(70,2)$ & $142(69,6)$ & $131(62,4)$ & NS \\
\hline $\begin{array}{l}\text { Prefiere alimentos con imágenes } 0 \\
\text { dibujos }\end{array}$ & $359(47,1)$ & $179(45,5)$ & $180(48,6)$ & NS & $160(45,6)$ & $95(46,8)$ & $104(49,8)$ & NS \\
\hline \multicolumn{9}{|l|}{ IMC materno aproximado $(\mathrm{N}=613)$} \\
\hline Sobrepeso (IMC: $25-29,9 \mathrm{~kg} / \mathrm{m}^{2}$ ) & $180(29,4)$ & $95(29,8)$ & $85(28,9)$ & NS & $78(27,7)$ & $56(33,7)$ & $46(27,9)$ & NS \\
\hline Obesidad (IMC $\geq 30$ kg/m²) & $134(21,9)$ & $65(20,4)$ & $69(23,5)$ & NS & $74(26,2)$ & $24(14,5)$ & $36(21,8)$ & NS \\
\hline
\end{tabular}


Tabla 5. Sobrepeso y obesidad en niños de nivel 5 de Montevideo. Relación con número de personas en el hogar, alimentación, actividad física del niño, y sobrepeso u obesidad materna.

\begin{tabular}{|c|c|c|c|c|c|c|c|c|c|}
\hline & \multicolumn{3}{|c|}{ Sobrepeso u obesidad } & \multicolumn{3}{|c|}{ Obesidad } & \multicolumn{3}{|c|}{ Obesidad abdominal } \\
\hline & $\%$ & $\mathrm{RR}$ & $p$ & $\%$ & $\mathrm{RR}$ & $p$ & $\%$ & $\mathrm{RR}$ & $p$ \\
\hline \multicolumn{10}{|l|}{ Personas en el hogar } \\
\hline$\geq 5$ personas & 38,7 & 1 & & 13,3 & 1 & & 15,5 & 1 & \\
\hline$\leq 4$ personas & 43,6 & - & NS & 19,3 & $1,4(1,03-2,0)$ & 0,04 & 11,4 & - & NS \\
\hline \multicolumn{10}{|l|}{ Actividad física diaria } \\
\hline$\geq 60$ min por día & 41,4 & 1 & & 15,3 & 1 & & 13,2 & 1 & \\
\hline$<60$ min por día & 39,2 & - & NS & 17,3 & - & NS & 14,1 & - & NS \\
\hline \multicolumn{10}{|l|}{ Horas de sueño nocturno } \\
\hline Cumple con recomendación & 39,8 & 1 & & 15,8 & 1 & & 12,4 & 1 & \\
\hline No cumple con recomendación & 42,8 & - & NS & 18,0 & - & NS & 13,9 & - & NS \\
\hline \multicolumn{10}{|l|}{ Horas de pantalla al día } \\
\hline No supera recomendación & 35,9 & 1 & & 13,5 & 1 & & 10,2 & 1 & \\
\hline Supera recomendación & 43,4 & - & NS & 18,2 & - & NS & 14,4 & - & NS \\
\hline \multicolumn{10}{|l|}{ Desayuno } \\
\hline Sí & 41,2 & 1 & & 13,6 & 1 & & 13,8 & 1 & \\
\hline No & 39,7 & - & NS & 16,8 & - & NS & 13,4 & - & NS \\
\hline \multicolumn{10}{|c|}{ Alfajores, obleas o bizcochos en el desayuno } \\
\hline No consume & 38,2 & 1 & & 13,6 & 1 & & 12,4 & 1 & \\
\hline Sí consume & 43,8 & - & NS & 19,7 & $1,5(1,06-2,3)$ & 0,02 & 14,5 & - & NS \\
\hline \multicolumn{10}{|l|}{ Consumo de frutas } \\
\hline Todos los días & 39,3 & 1 & & 15,9 & 1 & & 12,3 & 1 & \\
\hline No consumo diario & 42,6 & - & NS & 17,1 & - & NS & 14,5 & - & NS \\
\hline \multicolumn{10}{|l|}{ Consumo de verduras } \\
\hline Todos los días & 39,0 & 1 & & 15,8 & 1 & & 11,2 & 1 & \\
\hline No consumo diario & 41,7 & - & NS & 16,7 & - & NS & 14,2 & - & NS \\
\hline \multicolumn{10}{|l|}{ Consumo de refrescos } \\
\hline$\leq 2$ dias/semana & 40,1 & 1 & & 16,7 & 1 & & 11,9 & 1 & \\
\hline$\geq 3$ días/semana & 41,1 & - & NS & 16,2 & - & NS & 14,6 & - & NS \\
\hline \multicolumn{10}{|c|}{ Consumo nuggets, "panchos", fiambres } \\
\hline$\leq 2$ días/semana & 37,6 & 1 & & 11,8 & 1 & & 8,1 & 1 & \\
\hline$\geq 3$ días/semana & 42,4 & - & NS & 18,5 & $1,6(1,05-2,3)$ & & 15,7 & $1,91,2-3,1)$ & \\
\hline \multicolumn{10}{|l|}{ Alimentos que adjuntan juguetes } \\
\hline Nunca, rara vez, a veces los prefiere & 38,6 & 1 & & 12,4 & & & 11,6 & 1 & \\
\hline Los prefiere habitualmente & 42,0 & - & NS & 18,4 & $1,5(1,01-2,1)$ & 0,03 & 14,3 & - & NS \\
\hline \multicolumn{10}{|l|}{ IMC de la madre } \\
\hline Normopeso & 34,4 & 1 & & 12,2 & 1 & & 10,3 & 1 & \\
\hline Sobrepeso u obesidad & 47,4 & $1,7(1,2-2,4)$ & 0,00 & 21,2 & $1,9(1,2-3,0)$ & 0,00 & 16,7 & $1,7(1,1-2,8)$ & 0,02 \\
\hline Obesidad de la madre & 50,0 & $1,6(1,1-2,3)$ & 0,00 & 23,3 & $1,7(1,1-2,8)$ & & 20,9 & $2,0(1,2-3,3)$ & \\
\hline
\end{tabular}


Tabla 6. Sobrepeso y obesidad en niños de nivel 5 de Montevideo. Presión arterial periférica (braquial) según sobrepeso, obesidad; obesidad abdominal $(n=575)$.

\begin{tabular}{|c|c|c|c|c|c|}
\hline & $\begin{array}{l}\text { Total }^{*} \\
(n=575)\end{array}$ & $\begin{array}{l}\text { Sobrepeso } \\
(n=242)\end{array}$ & $\begin{array}{l}\text { Obesidad } \\
(n=107)\end{array}$ & $\begin{array}{l}\text { Obesidad abdominal } \\
\qquad(n=84)\end{array}$ & $p$ \\
\hline \multicolumn{6}{|l|}{ PAS (mm Hg) } \\
\hline $\bar{x} \pm D E$ & $99 \pm 7$ & $101 \pm 6$ & $103 \pm 5$ & $102 \pm 5$ & $<0,01$ \\
\hline Percentil medio & 60 & 65 & 70 & 69 & $<0,01$ \\
\hline \multicolumn{6}{|l|}{ PAD (mm Hg) } \\
\hline $\bar{x} \pm D E$ & $59 \pm 5$ & $60 \pm 4$ & $61 \pm 5$ & $69 \pm 21$ & $<0,01$ \\
\hline Percentil medio** & 62 & 67 & 70 & 70 & $<0,01$ \\
\hline
\end{tabular}

pediátrica ${ }^{(29)}$. En el presente estudio no se observaron niños hipertensos, probablemente por su corta edad. Sin embargo, la PA periférica (braquial), tanto sistólica como diastólica, resultaron más elevadas que las esperadas según el sexo, especialmente en los niños con sobrepeso y obesidad. La tendencia del sobrepeso y obesidad a presentar mayores niveles de PA se ha asociado con cambios en el volumen sistólico o en la velocidad de la onda de pulso aórtica ${ }^{(6)}$. Mediante evaluación cardiovascular no invasiva se ha demostrado que independientemente de la edad y sexo, los niños y adolescentes con sobrepeso u obesidad asocian cambios en parámetros hemodinámicos y de estructura y función vascular ${ }^{(30)}$. Estos cambios se instalan precozmente y son potencialmente reversibles si se interviene en forma oportuna.

El sesgo de información, relacionado con la utilización de una entrevista estructurada para recabar información relacionada con posibles factores de riesgo, en especial el IMC materno mediante autorreporte de sus datos antropométricos, constituye la principal limitante.

A pesar de ello, los resultados de este estudio ponen en evidencia el potencial "ambiente obesogénico" en el que se desarrolla la vida de niños y niñas. Éste se caracteriza no solo por madre obesa, sino también por el elevado consumo de productos de bajo valor nutricional con alto contenido en azúcar, grasa y sal, elevado consumo de bebidas azucaradas, excesiva exposición a pantallas, o insuficientes horas diarias de actividad física y de sueño ${ }^{(12,31-40)}$. En esta serie, más de la mitad de los niños evaluados tomaba refrescos o jugos y consumía alfajores, galletitas rellenas, obleas o bizcochos, adicionalmente un tercio de los niños consumía golosinas con una frecuencia $\geq 3$ días/semana. Además, casi el $30 \%$ consumía nuggets, "panchos" o fiambres con una frecuencia $\geq 3$ días/semana, lo que sugiere un elevado consumo de sodio, que se relacionaría con mayor riesgo de cifras elevadas de PA. En el presente trabajo los niños que consumían frecuentemente este tipo de alimentos aumentaron el riesgo de obesidad en una vez y media. Uruguay ha tenido, entre los países de América Latina, el más rápido crecimiento en la venta de alimentos ultraprocesados. En niños y adolescentes el consumo frecuente de estos productos se ha demostrado que está asociado con mayor masa grasa corporal y prevalencia de síndrome metabólico y dislipemia ${ }^{(41-44)}$.

Por otra parte, en los niños estudiados se observó un bajo consumo diario de frutas y verduras. Existe evidencia a favor de que el consumo diario de cantidades adecuadas de frutas y verduras se relaciona con menor IMC, menor adiposidad visceral y niveles más bajos de colesterol total, colesterol-LDL y $\mathrm{PA}^{(45,46)}$.

También se observó la fuerte influencia de la publicidad, ya que la mayoría de los niños recordaba mensajes de productos comestibles y casi siempre o siempre prefería los productos promocionados. Está demostrado que la publicidad y propaganda dirigida a los niños influye en sus preferencias y solicitudes de compra ${ }^{(42-49)}$. Se ha estimado que la contribución de la publicidad televisiva de alimentos a la prevalencia de la obesidad en niños de 6 a 11 años varía entre 16\%-40\% en Estados Unidos, 10\%-28\% en Australia e Italia y 4\%-18\% en Gran Bretaña, Suiza y Países Bajos ${ }^{(50)}$. En el presente trabajo los niños que preferían alimentos que adjuntan juguetes, una forma de publicidad y mercadeo, presentaron una predisposición una vez y media más elevada a presentar obesidad.

Además de los malos hábitos en la alimentación, se observó hábitos inadecuados respecto al sueño y la acti- 
vidad física. A pesar de que en este estudio no se observó asociación significativa, un tercio de los niños dormía menos de las horas recomendadas y el $60 \%$ resultó sedentario o poco activo (realizaba diariamente niveles muy leves o leves de actividad física ${ }^{(9,40)}$. Solo uno de cada cinco niños concurría a un centro deportivo y un cuarto nunca realizaba actividad física en sus momentos de ocio. Agravando la inactividad física, dos tercios de los niños permanecían por un tiempo $\geq 2,5$ horas frente a pantallas. Las últimas recomendaciones de la Academia Americana de Pediatría para los niños de 2 a 5 años son de limitar el uso de pantallas a $\leq 1$ hora por día. A partir de los 6 años en adelante se recomienda buscar un equilibrio entre el juego con medios digitales y el juego dentro de la vida cotidiana. La exposición frente a las pantallas puede afectar el sueño, la actividad física y la comunicación con otros niños y adultos ${ }^{(10,11,51)}$. Estos parámetros forman también parte del denominado "ambiente obesogénico".

En este estudio no se halló asociación entre el exceso de peso en los niños y el máximo nivel de educación formal alcanzado por las madres o el nivel de ingreso económico del hogar. En una encuesta realizada en 2007 por Amarante y colaboradores se había observado que a medida que aumentaban los ingresos se incrementaba la prevalencia de sobrepeso y obesidad infantil ${ }^{(27)}$. En los países desarrollados se ha observado una asociación inversa entre nivel el socioeconómico y la obesidad infantil, mientras que en los países en desarrollo la obesidad es más frecuente en familias de mayor nivel socioeconómico $^{(34)}$. Por otra parte, en el estudio ALADINO se reportó mayor prevalencia de obesidad en hijos de padres con estudios primarios y secundarios y mayor porcentaje de niños con peso adecuado entre los hijos de aquellos con estudios universitarios ${ }^{(21)}$. Sin embargo, en la mayoría de los países de América Latina la prevalencia de sobrepeso y obesidad en niños en edad preescolar aumenta en hijos de madres con educación superior ${ }^{(23,24,52,53)}$. En este trabajo la mayoría de los niños eran hijos de madres sin estudios universitarios.

Resulta necesario sensibilizar a los padres, profesionales de la salud y educadores sobre los hallazgos de este estudio y promover que los tomadores de decisiones desarrollen estrategias intersectoriales que contribuyan a reorientar las políticas públicas para prevenir la obesidad desde etapas muy tempranas.

\section{Conclusiones}

Entre 2016 y 2017 la prevalencia de sobrepeso u obesidad y obesidad en niños de nivel 5 que asistieron a jardines públicos de Montevideo fue de 40,6\% y 16,5\%, respectivamente. Este hallazgo confirma la tendencia creciente de este problema de salud pública. Los hogares con menos de cuatro personas, la obesidad materna según IMC aproximado, la preferencia de alimentos que adjuntan juguetes y el consumo frecuente de embutidos en la semana y de alfajores, obleas o bizcochos en el desayuno, aumentó el riesgo de obesidad infantil una vez y media. Los hábitos de alimentación, actividad física y sueño observados completan el "ambiente obesogénico" en que se desarrollan estos niños. Es preocupante su repercusión temprana sobre la PA.

\section{Agradecimientos}

A todos los niños, niñas y familias que aceptaron ser parte del estudio. A las autoridades de la educación y de cada centro educativo. A la Agencia Nacional de Investigación e Innovación (ANII; FSPI_X_2015_1_108509 y FSPI_X_2015_1_108484). A los integrantes de los equipos de trabajo que contribuyeron con determinaciones de las variables incluidas. En los registros de presión arterial participaron Br. Mariana Marin, Br. Agustina Zinoveev, Br. Juan M. Castro, Dra. Victoria García-Espinosa.

\section{Summary}

Introduction: in recent years, Uruguay has seen an increase of the prevalence of overweight and obesity. Scientific evidence has shown that early childhood conditions are determinant.

Objective: to determine the prevalence of overweight, obesity and brachial blood pressure levels and identify associated risk factors in 5-year-old children who attend public preschool centers in Montevideo.

Method: transversal study (7/2016-6/2017) in a representative sample of 7715 -year-old children from preschool centers in Montevideo. We assessed their history, eating habits, physical activity, sleeping habits, weight and maternal size. We recorded anthropometry and brachial blood pressure for each child.

Results: the prevalence of overweight or obesity was $40.6 \%$ (CI 95\%: 37.4- 44.3), obesity $16.5 \%$ (CI 95\%: 13.9-19.1) and abdominal obesity $12.9 \%$ (CI 95\%: $11.0-15.8$ ). $29.4 \%$ of mothers reported overweight and $21.9 \%$ of them reported obesity. Sedentary lifestyle was found in $58.3 \%$, long screen viewing in $60.4 \%$ and inadequate sleep in $40.9 \%$. Frequent consumption of pastries, wafers and biscuits for breakfast, cold cuts during week days, preference of food they see in advertisements, and reported maternal overweight or obesity were associated to child obesity. Children with overweight and/or abdominal obesity evidenced higher levels of brachial blood pressure.

Conclusions. prevalence of overweight and obesity in 5-year-old children from public preschool centers in Montevideo is high. Maternal overweight and obesity 
and habits in connection with eating, physical activity and amount of sleep are associated with this condition.

\section{Resumo}

Introdução: nos últimos anos observou-se um aumento da prevalência de sobrepeso e obesidade no Uruguai. A evidência científica mostra que as condições de vida na primeira infância são determinantes.

Objetivo: determinar a prevalência de sobrepeso, obesidade e os niveles de pressão arterial (PA) braquial e identificar fatores de risco associados em crianças com 5 anos que frequentam jardines de infância públicos em Montevidéu.

Métodos: estudo transversal (7/2016-6/2017) em uma amostra representativa de 771 crianças com 5 anos de jardins de infância públicos de Montevidéu. Foram coletados dados sobre a história e o comportamento alimentar, atividade física, hábitos de sono e peso e altura materna. Os dados antropométricos e PA braquial de todas as crianças foram registrados.

Resultados: a prevalência de sobrepeso ou obesidade foi de 40,6\% (I.C.95\%:37,4-44,3), obesidade 16,5\% (I.C.95\%:13,9-19,1) e obesidade abdominal 12,9\% (I.C.95\%:11,0-15,8). 29,4\% das mães informaram sobrepeso e $21,9 \%$ obesidade. Observou-se sedentarismo em $58,3 \%$, exposição prolongada a telas de equipamentos eletrônicos em $60,4 \%$ e poucas horas de sono em $40,9 \%$. O consumo frequente de alfajores, obleias e pães com altos teores de açúcar e gordura no café da manhã, de embutidos nos dias de semana, preferência de alimentos com publicidade, e a informação de sobrepeso ou obesidade materna estava associado a obesidade infantil. As crianças com sobrepeso, obesidade e/ou obesidade abdominal apresentaram niveles mais altos de PA braquial.

Conclusões: a prevalência de sobrepeso e obesidade em crianças com 5anos de jardins de infância públicos de Montevidéu é elevada. O sobrepeso e obesidade materna e os hábitos relacionados à alimentação, atividade física e sono, estão associados à sua presença.

\section{Bibliografía}

1. Uruguay. Ministerio de Salud Pública, Dirección General de Salud, División Epidemiología, Departamento de Vigilancia en Salud. $1^{\text {a }}$ Encuesta nacional de factores de riesgo de enfermedades crónicas no transmisibles. 2006. Disponible en: www.gub.uy/ministerio-salud-publica/comunicacion/publicaciones/primera-encuesta-nacional-de-factores-de-riesgo-de-enfermedades-cronicas [Consulta: 10 setiembre 2017].

2. Uruguay. Ministerio de Salud, Programa de Prevención de Enfermedades No Transmisibles. $2^{\text {a }}$ Encuesta nacional de factores de riesgo y enfermedades crónicas no transmisibles. 2013.Disponible en: www.gub.uy/ministerio-salud-pu- blica/comunicacion/publicaciones/2da-encuesta-nacional-de-factores-de-riesgo-de-enfermedades-no [Consulta: 15 setiembre 2017].

3. Gluckman PD, Hanson MA, Cooper C, Thornburg KL. Effect of in utero and early-life conditions on adult health and disease. N Engl J Med 2008; 359(1):61-73.

4. Koskinen J, Magnussen C, Sinaiko A, Woo J, Urbina E, Jacobs D, et al. Childhood age and associations between childhood metabolic syndrome and adult risk for metabolic syndrome, Type 2 diabetes mellitus and carotid intima media thickness: The International Childhood Cardiovascular Cohort Consortium. J Am Heart Assoc 2017; 6(8):1-16.

5. Friend A, Craig L, Turner S. The prevalence of metabolic syndrome in children: a systematic review of the literature. Metab Syndr Relat Disord 2013; 11(2):71-80.

6. García-Espinosa V, Curcio S, Castro JM, Arana M, Giachetto $\mathbf{G}$, et al. Children and adolescent obesity associates with pressure-dependent and age-related increase in carotid and femoral arteries' stiffness and not in brachial artery, indicative of nonintrinsic arterial wall alteration. Int J Vasc Med 2016; 2016:4916246.

7. Estragó V, Álvarez Vaz R, Tabárez A, Bulla D, Díaz J, Zelarayán M. Sobrepeso, obesidad e hipertensión arterial en niños, una aproximación al problema. Arch Pediatr Urug 2018; 89(5):301-10

8. Nascimento Ferreira MV, Ferreira De Moraes AC, Toazza-Oliveira PV, Forjaz CLM, Aristizabal JC, Santaliesra-Pasías $\mathbf{A M}$, et al. Reliability and validity of a questionnaire for physical activity assessment in South American children and adolescents: the SAYCARE Study. Obesity 2018; 26(Suppl 1):S23-S30.

9. Organización Mundial de la Salud. Recomendaciones mundiales sobre actividad física para la salud. Ginebra: OMS, 2010. Disponible en: http://apps.who.int/iris/bitstream/10665/44441/1/9789243599977_spa.pdf [Consulta: 10 setiembre 2017].

10. Council on Communications and Media. Media and young minds. Pediatrics 2016; 138(5):e20162591.

11. Council on Communications and Media. Media use in school-aged children and adolescents. Pediatrics 2016; 138(5):e20162592.

12. Paruthi S, Brooks LJ, D'Ambrosio C, Hall WA, Kotagal S, Lloyd RM, et al. Recommended amount of sleep for pediatric populations: a consensus statement of the American Academy of Sleep Medicine. J Clin Sleep Med 2016; 12(6):785-6.

13. Physical status: the use and interpretation of anthropometry. Report of a WHO Expert Committee. World Health Organ Tech Rep Ser 1995; 854:1-452.

14. Girona A, Iturralde A, Köncke F, Pandolfo V. Evaluación antropométrica desde el nacimiento hasta los 5 años. Material de apoyo técnico para los servicios de salud. Montevideo: MSP-MIDES, 2016. Disponible en: guiaderecursos.mides.gub.uy/innovaportal/file/63323/1/rotafolio-guia-antropometrica.pdf [Consulta: 3 setiembre 2017]. 
15. Organización Mundial de la Salud. Guía para las mediciones físicas (Step 2). En: Manual de vigilancia STEPS de la OMS: el método STEPwise de la OMS para la vigilancia de los factores de riesgo de las enfermedades crónicas. Ginebra: OMS, 2006. Disponible en: www.who.int/chp/steps/Parte3_Seccion4.pdf [Consulta: 24 setiembre 2017].

16. Fernández JR, Redden DT, Pietrobelli A, Allison DB. Waist circumference percentiles in nationally representative samples of African-American, European-American, and Mexican-American children and adolescents. J Pediatr 2004; 145(4):439-44.

17. Mancia G, Fagard R, Narkiewicz K, Redón J, Zanchetti A, Bohm M, et al. 2013 ESH/ESC Guidelines for the management of arterial hypertension: the task force for the management of arterial hypertension of the European Society of Hypertension (ESH) and of the European Society of Cardiology (ESC). J Hypertens 2013; 31(7):1281-357.

18. Lurbe E, Agabiti-Roseic E, Cruickshankd JK, Dominiczake A, Erdinef S, Hirthg A, et al. 2016 European Society of Hypertension guidelines for the management of high blood pressure in children and adolescents. J Hypertens_2016; 34(10):1887-920.

19. Organización Mundial de la Salud. Obesidad y sobrepeso. 2017. Disponible en: www.who.int/es/news-room/fact-sheets/ detail/obesity-and-overweight [Consulta: 24 setiembre 2017].

20. Ahrens W, Pigeot I, Pohlabeln H, De Henauw S, Lissner L, Molnár D, et al. Prevalence of overweight and obesity in European children below the age of 10. Int J Obes (Lond) 2014; 38(Suppl 2):S99-107.

21. Estudio ALADINO 2015. Estudio de vigilancia del crecimiento, alimentación, actividad física, desarrollo infantil y obesidad en España 2015. Madrid: Ministerio de Sanidad, Servicios Sociales e Igualdad. Agencia Española de Consumo, Seguridad Alimentaria y Nutrición, 2016. Disponible en: http://www.aecosan.msssi.gob.es/AECOSAN/docs/documentos/nutricion/observatorio/Estu-

dio_ALADINO_2015.pdf [Consulta: 15 octubre 2017].

22. Corvalán C, Garmendia ML, Jones-Smith J, Lutter CK, Miranda JJ, Pedraza LS, et al. Nutrition status of children in Latin America. Obes Rev 2017; 18(Suppl 2):7-18.

23. Rivera JÁ, de Cossío TG, Pedraza LS, Aburto TC, Sánchez TG, Martorell R. Childhood and adolescent overweight and obesity in Latin America: a systematic review. Lancet Diabetes Endocrinol 2014; 2(4):321-32.

24. Brasil. Ministério da Saúde, Instituto Brasileiro de Geografia e Estatística, Ministério do Planejamento, Orçamento e Gestão. Pesquisa de orçamentos familiares 2008-2009. Antropometria e estado nutricional de crianças, adolescentes e adultos no Brasil. Rio de Janeiro: IBGE, 2010. Disponible en: biblioteca.ibge.gov.br/visualizacao/livros/liv45419.pdf [Consulta: 10 octubre 2017].

25. Chile. Junta Nacional de Auxilio Escolar y Becas. Departamento de Planificación, Control de Gestión y Estudios. Informe Mapa Nutricional 2015: situación nutricional de los preescolares y escolares de establecimientos municipalizados y particulares subvencionados del país. JUNAEB, 2016. Dis- ponible en: www.junaeb.cl/wp-content/uploads/2017/07/ Informe-Mapa-Nutricional-2015.pdf [Consulta: 2 noviembre 2017].

26. Pisabarro R, Recalde A, Irrazábal E, Chaftare Y. ENSO niños 1: primera encuesta nacional de sobrepeso y obesidad en niños uruguayos. Rev Méd Urug 2002; 18(3):244-50.

27. Amarante V, Arim R, Severi C, Vigorito A, Aldabe I. El estado nutricional de los niños/as y las políticas alimentarias. Resultados de una encuesta sobre situación nutricional en escolares de primer año. Montevideo: Universidad de la República, UNICEF, Cooperazione Italiana, PNUD, 2007. Disponible en: www.infamilia.gub.uy/pageredirect.aspx?0,292 [Consulta: 2 noviembre 2017].

28. Manios Y, Moschonis G, Karatzi K, Androutsos O, Chinapaw M, Moreno LA, et al. Large proportions of overweight and obese children, as well as their parents, underestimate children's weight status across Europe. The ENERGY (EuropeaN Energy balance research to prevent excessive weight gain among youth) project. Public Health Nutr 2015; 18(12):2183-90.

29. Ayer J, Charakida M, Deanfield JE, Celermajer DS. Lifetime risk: childhood obesity and cardiovascular risk. Eur Heart J 2015; 36(22):1371-6.

30. García-Espinosa V, Bia D, Castro J, Zinoveev A, Marin M, Giachetto G, et al. Peripheral and central aortic pressure, wave-derived reflection parameters, local and regional arterial stiffness and structural parameters in children and adolescents: impact of body mass index variations. High Blood Press Cardiovasc Prev 2018; 25(3):267-80.

31. Bove MI, Cerruti F. Encuesta de lactancia, estado nutricional y alimentación complementaria en niños menores de 24 meses atendidos por servicios públicos y mutuales de Montevideo y el interior del país. Montevideo: UNICEF, RUANDI, MSP, 2007:72 p. Disponible en: http://dspace.mides.gub.uy:8080/xmlui/bitstream/handle/123456789/

264/UNICEF\%20-\%20Encuesta\%20de\%20lactancia $\% 2 \mathrm{c} \% 20$ estado $\% 20$ nutricionaly $\% 20$ alimentacion $\% 20$ complementaria.pdf? sequence $=1 \&$ is Allowed $=y$ [Consulta: 22 octubre 2017].

32. Jiménez-Cruz A, Wojcicki JM, Bacardí-Gascón M, Castellón-Zaragoza A, García-Gallardo JL, Schwartz N, et al. Maternal BMI and migration status as predictors of childhood obesity in Mexico. Nutr Hosp 2011; 26(1):187-93.

33. Aranceta-Bartrina J, Pérez-Rodrigo C. Determinants of childhood obesity: ANIBES study. Nutr Hosp 2016; 33(Supp14):339.

34. Wijnhoven TM, van Raaij JM, Yngve A, Sjoberg A, Kunesová M, Duleva V, et al. WHO European childhood obesity surveillance initiative: health-risk behaviours on nutrition and physical activity in 6-9-year-old schoolchildren. Public Health Nutr 2015; 18(17):3108-24.

35. Borges MC, Louzada ML, de Sá TH, Laverty AA, Parra DC, Garzillo JM, et al. Artificially sweetened beverages and the response to the global obesity crisis. PloS Med 2017; 14(1):e1002195. 
36. Monteiro CA, Gomes FS, Cannon G. The snack attack. Am J Public Health 2010; 100(6):975-81.

37. Herrera JC, Lira M, Kain J. Vulnerabilidad socioeconómica y obesidad en escolares chilenos de primero básico: comparación entre los años 2009 y 2013. Rev Chil Pediatr 2017; 88(6):736-43.

38. Louzada ML, Baraldi LG, Steele EM, Martinez E, Martins AP, Canella DS, et al. Consumption of ultra-processed foods and obesity in Brazilian adolescents and adults. Prev Med 2015; 81:9-15.

39. Wang F, Liu H, Wan Y, Li J, Chen Y, Zheng J, et al. Sleep duration and overweight/obesity in preschool-aged children: a prospective study of up to 48,922 children of the Jiaxing Birth Cohort. Sleep 2016; 39(11):2013-9.

40. Durán S, Haro P. Asociación entre cantidad de sueño y obesidad en escolares chilenos. Arch Arg Pediatr 2016; 114(2):114-9.

41. Perez-Morales E, Bacardí-Gascón M, Jiménez-Cruz A. Sugar sweetened beverage intake before 6 years of age and weight or BMI status among older children; systematic review of prospective studies. Nutr Hosp 2013; 28(1):47-51.

42. Moubarac JC, Martins AP, Claro RM, Levy RB, Cannon G, Monteiro CA, et al. Consumption of ultra-processed foods and likely impact on human health. Evidence from $\mathrm{Ca}$ nada. Public Health Nutr 2013; 16(12):2240-8.

43. Canella DS, Levy RB, Martins AP, Claro RM, Moubarac JC, Baraldi LG, et al. Ultra-processed food products and obesity in Brazilian households (2008-2009). PLoS One 2014; 9(3):e92752.

44. Monteiro CA, Moubarac JC, Levy RB, Canella DS, Louzada MLDC, Cannon G. Household availability of ultra-processed foods and obesity in nineteen European countries. Public Health Nutr 2018; 21(1):18-26.

45. Bradlee ML, Singer MR, Qureshi MM, Moore LL. Food group intake and central obesity among children and adolescents in the Third National Health and Nutrition Examination Survey (NHANES III). Public Health Nutr 2010; 13:797-805.

46. Moore LL, Singer MR, Qureshi MM, Bradlee ML. Dairy intake and anthropometric measures of body fat among chil- dren and adolescents in NHANES. J Am Coll Nutr 2008; 27:702-10

47. Moubarac JC, Parra DC, Cannon G, Monteiro CA. Food classification systems based on food processing: significance and implications for policies and actions: a systematic literature review and assessment. Curr Obes Rep 2014; 3(2): 256-72.

48. Organización Panamericana de la Salud. Alimentos y bebidas ultraprocesados en América Latina: tendencias, efecto sobre la obesidad e implicaciones para las políticas públicas. Washington, DC: OPS, 2015. Disponible en http://iris.paho.org/xmlui/bitstream/handle/123456789/7698/9789275318645_esp.pdf [Consulta: 15 setiembre 2017].

49. Organización Panamericana de la Salud. Recomendaciones de la consulta de expertos de la Organización Panamericana de la Salud sobre la promoción y publicidad de alimentos y bebidas no alcohólicas dirigidas a los niños en la Región de las Américas. Washington, DC: OPS, 2011. Disponible en: https://www.paho.org/hq/dmdocuments/2012/ExpertsFood-Marketing-to-Children-(SPA).pdf [Consulta: 24 setiembre 2017].

50. Goris JM, Petersen S, Stamatakis E, Veerman JL. Television food advertising and the prevalence of childhood overweight and obesity: a multicountry comparison. Public Health Nutr 2010; 13(7):1003-12.

51. Ren H, Zhou Z, Liu WK, Wang X, Yin Z. Excessive homework, inadequate sleep, physical inactivity and screen viewing time are major contributors to high paediatric obesity. Acta Paediatr 2017; 106(1):120-7.

52. Organización de las Naciones Unidas para la Alimentación y la Agricultura. Organización Panamericana de la Salud. América Latina y el Caribe. Panorama de la seguridad alimentaria y nutricional: sistemas alimentarios sostenibles para poner fin al hambre y la malnutrición. Santiago, 2017. Disponible en: http://www.fao.org/3/a-i6747s.pdf [Consulta: 3 noviembre 2017].

53. Salahuddin M, Pérez A, Ranjit N, Kelder SH, Barlow SE, Pont SJ, et al. Predictors of severe obesity in low-income, predominantly hispanic/latino children: the texas childhood obesity research demonstration study. Prev Chronic Dis 2017; 14:170129.

\footnotetext{
María Isabel Bove, https://orcid.org/0000-0001-9107-5355

Gustavo Giachetto, https://orcid.org/0000-0003-3775-4773

Raúl Ramírez, https://orcid.org/0000-0002-4175-8972

Caren Zelmonovich, https://orcid.org/0000-0002-7229-2327

Valentina Guillermo, https://orcid.org/0000-0003-4309-7335

Leticia Klaps, https://orcid.org/0000-0002-4204-0145

Adriana Iturralde, https://orcid.org/0000-0003-1609-5481

Fabiana Peregalli, https:/orcid.org/0000-0001-8961-4858

Daniel Bia, https://orcid.org/0000-0001-7160-7166

Yanina Zócalo, https://orcid.org/0000-0002-6544-2518
} 